\title{
Blockchain Utilization in Healthcare: Key Requirements and Challenges
}

\author{
Tanesh Kumar ${ }^{1}$, Vidhya Ramani ${ }^{1}$, Ijaz Ahmad ${ }^{1}$, An Braeken $^{2}$, Erkki Harjula ${ }^{1}$, Mika Ylianttila ${ }^{1}$ \\ ${ }^{1}$ Centre for Wireless Communication (CWC), University of Oulu, Finland \\ ${ }^{2}$ Industrial Engineering INDI, Vrije Universiteit Brussel VUB, Nijverheidskaai 170, 1070 Brussel \\ Email: ${ }^{1}$ [tanesh.kumar, vidhya.ramani, ijaz.ahmad, erkki.harjula, mika.ylianttila]@ oulu.fi, ${ }^{2}$ an.braeken@ vub.ac.be,
}

\begin{abstract}
Blockchain is so far well-known for its potential applications in financial and banking sectors. However, blockchain as a decentralized and distributed technology can be utilized as a powerful tool for immense daily life applications. Healthcare is one of the prominent applications area among others where blockchain is supposed to make a strong impact. It is generating wide range of opportunities and possibilities in current healthcare systems. Therefore, this paper is all about exploring the potential applications of blockchain technology in current healthcare systems and highlights the most important requirements to fulfill the need of such systems such as trustless and transparent healthcare systems. In addition, this work also presents the challenges and obstacles needed to resolve before the successful adoption of blockchain technology in healthcare systems. Furthermore, we introduce the smart contract for blockchain based healthcare systems which is key for defining the pre-defined agreements among various involved stakeholders.
\end{abstract}

Index Terms-Blockchain; Healthcare; Requirements and Challenges; Opportunities

\section{INTRODUCTION}

The research community has started to realize the potential of blockchain beyond the financial applications. Blockchain as a decentralized technology can be utilized in immense useful applications such as healthcare, logistics, supply chain management and Internet of Things (IoTs) among others [1], [2], [3]. Blockchain provides secure distributed database that can perform their tasks without any intervention of thirdparty or central administration. It is highly useful in the case when various relevant parties want to access same kind of the information. Thus, blockchain based systems have huge potential to minimize the cost and resources of current intermediates. Blockchain, generally uses cryptographic (such as hash functions, asymmetric encryption and digital signatures) based approaches among various stakeholders in a given system that helps in preserving trust among them while interaction.

Internet of things (IoTs) [4] and other communication related technologies (such as 5G) [5], [6] are putting a huge impact on the healthcare services and providing consumer better and improved medical services but provides an opportunity to the involved stakeholders to generate the revenue. Medical data storing and sharing is an integral part in healthcare systems in order to enhance the quality and services of overall healthcare systems. However, sharing data records among various entities through unsecure means can lead to leakage of the patient's personal and critical information. Also, the lack of the user control over their personal information can cause harmful consequences such as unauthorized entities can access the personal medical information. While sharing the patients information might be fragmented in various healthcare systems and that can lead to various risks [3].

One of the core issues in current electronic health/medical records (EHR/EMR) is maintaining the interoperability among various involved stakeholders [7]. This issue may cause obstacles in the data transaction among each other. The lack of coordinated data management and sharing mechanism among various entities may cause in fragmentation of the healthcare information. Apart from interoperability, data security and privacy are another key challenges in the current ways of data storing and sharing data through EHR/EMR systems [7], [9]. Most of the patients are hesitant about sharing and storing their personal medical information due to the data leakage and potential shortcoming in security mechanism [10], [11]. Therefore, there is a clear need of distributed way of data sharing and storing where patients are more sure about their data security and privacy and in addition all the involved stakeholders can see the holistic view of overall transaction and interactions [7].

Therefore, considering these challenges in the current healthcare systems, it is vital to utlilize the potential blockchain technology in the healthcare sector [3]. Blockchain technology can play a key role in number of healthcare applications, for example, using blockchain for electronic healthcare records (EHR/EMR) can ensure the security of the critical information of the patients and can make sure that only valid and authorized entities should access the data [8], [12], [13], [14]. Blockchain for healthcare also uses the concept of smart contracts which enables trustless features among various entities in the system. Smart contracts consist of computer program that presents the agreements and principles on which all the involved parties in the network agrees upon and therefore no trusted third party is required [15], [16]. Blockchain based healthcare system are also proposed in a way that they support the interoperability feature in the system.

Hence, the main aim of this work is to overview and explore the blockchain technology for the purpose of healthcare based applications. In addition to that, this paper discusses the potential requirement and challenges for building blockchain based healthcare systems. Furthermore, we will also discuss the the need of the smart contracts for the blockchain based healthcare systems. The rest of the paper is organized as follows: Section 
II defines the opportunities of the blockchain in healthcare systems and section III highlights the key requirements needed for building such systems. Section IV discusses the potential challenges and obstacles in the way of blockchain helathcare systems. Section V presents the use of smart contracts for blockchain based healthcare systems. Section VI provides the discussion and future scope and we conclude in section VII.

\section{BlockChain Applications in HealthCARE INDUSTRY}

Blockchain as a decentralized and distributed technology has enormous applications in healthcare domain. Blockchain technology mainly introduced for the banking and finance sector, but now intended to provide safer ways of data sharing between providers, payers and patients in the healthcare systems. Due to immense potential and realistic applicability of blockchain in healthcare system, researchers has started to explore potential use cases for blockchain in healthcare industry. We briefly discuss few of them below as shown in Figure 1 and Table I:

\section{A. Clinical Data Sharing}

A crucial and key application of blockchain in healthcare is the sharing of medical data among various entities in the system. EHRs and EMRs contain highly critical and sensitive medical information related to the patient which need to be securely stored, shared, processed and accessed. Thus, in order to improve and enhance the quality of healthcare services, the medical information need to be stored and shared frequently among various relevant participants such as patients, doctors, healthcare service providers, pharmacies, insurance companies and researchers among others. In general, these kind of critical data sharing require strong measures for transparency and accountability while data transactions. Therefore, blockchain adds more transparency in such cases as it maintains a distributed ledger among all involved entities within the network. The blockchain provides a trustable and secure way of data sharing and management mechanisms where all parties are aware of transactions [14], [17], [18], [19].

\section{B. Global Data Sharing}

There are also certain occasions, where patients travel outside their own country for tourism purposes or for any other reasons. There are also situations when they may require to consult with a doctor for treatment of any disease. In that case, in order to provide better health services, respective doctors/hospitals of the other country should have knowledge of the patient healthcare information. Through blockchain based approaches, the medical information can easily be shared with the required entities residing in the other country and the patient would be consent and have control over the data. In order to receive a better medical treatment outside the country, the medical history of the respective patient should be known for example, the respective patient has any kind of allergy to some medicines or knowledge of his/her recent treatment. Thus the medical history needs to be securely accessed by the service provider [3], [18].

\section{Maintaining Medical History:}

Blockchain technology can also be well-utilized to store and maintain the medical history of the patients. For example, sometimes patients visit disconnected hospitals and thus the overall chain of the medical history might not be available

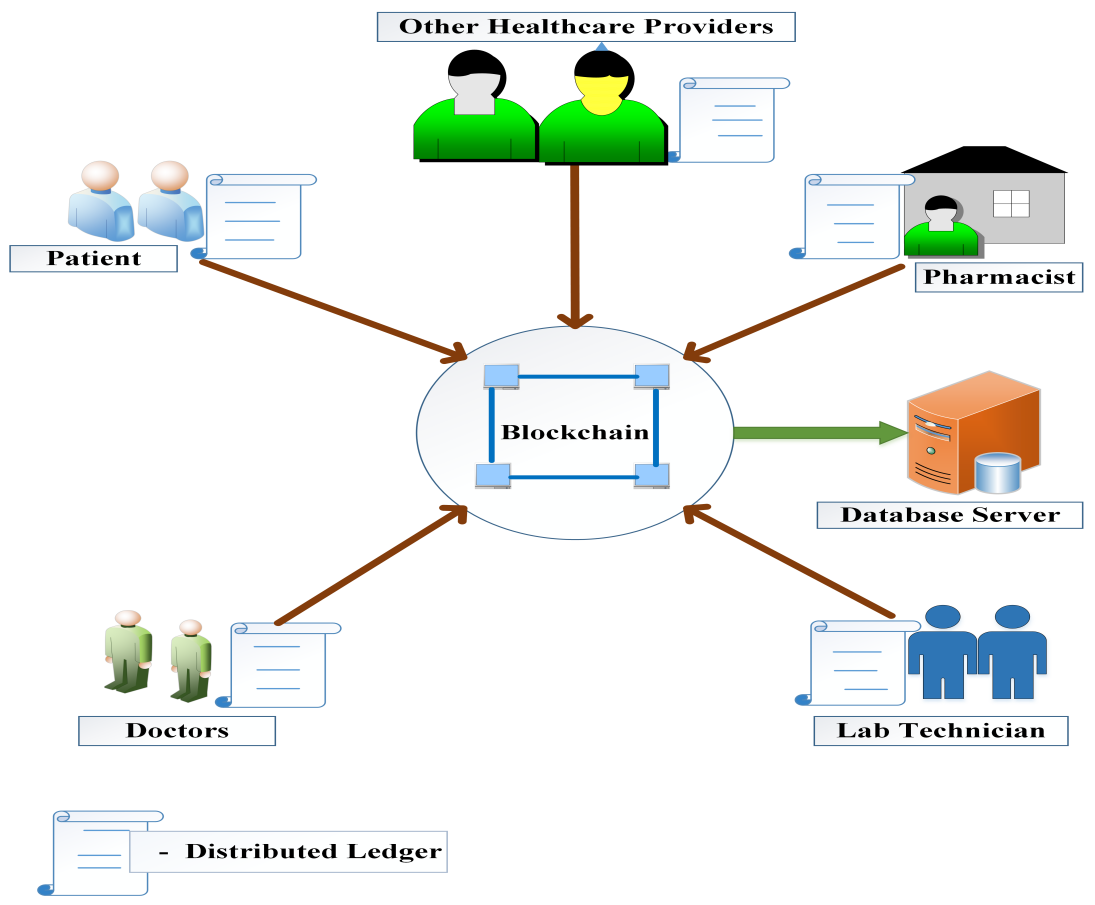

Fig. 1: Blockchain utilization in various healthcare applications. 
TABLE I: Various applications areas of blockchain based healthcare systems

\begin{tabular}{|l|l|l|}
\hline Application Area & Target Research Challenge & Description \\
\hline \hline Clinical data sharing & Secure data accessibility & $\begin{array}{l}\text { It must ensures secure medical data storing and sharing among } \\
\text { various involved stakeholders. }\end{array}$ \\
\hline Global data sharing & Securely global data storing and sharing & $\begin{array}{l}\text { It should provides secure healthcare data even outside the respec- } \\
\text { tive country./from anywhere in the world at global level. }\end{array}$ \\
\hline Maintaining Medical History & Availability of medical data or records & $\begin{array}{l}\text { It should guarantee for continuous availability of medical data to } \\
\text { maintain the medical record for better treatment and avoid extra } \\
\text { resources and costs. }\end{array}$ \\
\hline Research and Clinical Trials & traceability of trial phases & $\begin{array}{l}\text { It can be useful tool to provide the secure traceability for crucial } \\
\text { research and clinical trials. }\end{array}$ \\
\hline Healthcare Data Access Control & Managing access control & $\begin{array}{l}\text { It promises to give patients more secure access control to manage } \\
\text { their healthcare data. }\end{array}$ \\
\hline $\begin{array}{l}\text { Drug Supply Chain Manage- } \\
\text { ment }\end{array}$ & $\begin{array}{l}\text { Counterfeiters and pilfering of supply } \\
\text { chain process }\end{array}$ & $\begin{array}{l}\text { It can provide secure means of handling and monitoring the supply } \\
\text { chain processes in healthcare systems. }\end{array}$ \\
\hline Billing/Payers & Delay/Fraud in paying bills & $\begin{array}{l}\text { It can provide blockchain based healthcare payment solutions } \\
\text { which will be secure, less complex and faster. }\end{array}$ \\
\hline
\end{tabular}

or well-maintained (because of discontinuity and unavailability of previous records). In order to overcome such issues, blockchain can be useful in maintaining the history of records for each visit to any hospital. In addition, due to unavailability of the some disconnected data related to medical/laboratory reports, the patients again have to repeat the same medical tests. It will not only increase the cost of repeating the same laboratory test, but it can also be risky to do some test with high radiations again and again [8], [20].

\section{Research and Clinical Trials:}

Clinical trials represent another key and valuable process in the healthcare sector that require appropriate monitoring at each stage of the trail. For example, collection of required data, trial mechanism and monitoring and data management of trails [21], [22], [23]. These trial stages will consume heaps of resources as multiple parties need to coordinate with each other. These phases of trails also required massive trust among the various involved entities. Therefore, the blockchain can be a vital tool to deal with such research trials where each phase can be properly traced and data can be managed and analyzed without much waste of resources.

\section{E. Healthcare Data Access Control:}

With the recent technological advancements users control/grip over their own data is becoming weak. Especially, for the healthcare data, users are mostly unaware regarding which entities access their medical data and for what purposes and whether they are authorized to access it. For example in the case of EHR/EMR, various healthcare service providers are associated and patients are not fully aware about the parties that are accessing, storage and sharing of their medical data. Blockchain technology can allow the patients not only access their medical information in a more secure and safer way but also ensure only authorized entities can access and alter the data [8], [20], [24].

\section{F. Drug Supply Chain Management:}

Medical drug supply management is crucial in the modern medicine industry but it still suffers from various complexities and losses because of counterfeiters and pilfering. Blockchain can keep the traceability of such supply chain operations and enhance the integrity of the overall process [18], [25], [26]. The blockchain will be helpful in verifying the authenticity of the drugs and its supply chain to the authorized parties. Thus, blockchain can be vital in monitoring the various phases in the drug supply chain management and give access to the valid users.

\section{G. Billing/Payers:}

Traditional modes of patient billing systems are considered to be very complex and are exposed to billing related frauds. In addition to this, the process in most of the cases take more resources and time to receive all the required bills. It is expected that blockchain based payment solutions will make the billing process much more easier as compared with traditional billing approaches, where it takes several days to claim the bills. Also in the case the insurance company is involved in the paying process, it even takes several weeks to claim the bills. Blockchain would be a useful tool in such scenarios, where the insurance company is there for the payment. In addition, this also makes the insurance claims faster and can decrease the extra resources, time and cost [26], [27].

\section{KEY REQUIREMENTS}

In this section, the core focus is to highlight the key requirements needed while proposing a blockchain based healthcare system as shown in Table II.

\section{A. Nationwide Interoperability:}

This is one of the key requirements for blockchain based healthcare systems. The current healthcare system lacks the nationwide interoperability which is considered as one of the 
TABLE II: Key requirements in blockchain based healthcare systems

\begin{tabular}{|l|l|l|}
\hline Requirements Type & Description & Relevant Literature \\
\hline \hline Nationwide Interoperability & To define universal standards for healthcare data interoperability & [7], [19], [20], [24] \\
\hline Data Security & Secure the data in transit and in storage systems & [7], [8], [14], [19], [28], [29] \\
\hline Data Integrity & To ensure the healthcare data is consistent throughout the process & {$[14][19],[20],[29]$} \\
\hline Cost/Resources Effectiveness & Shared processing for higher and enhanced performance & {$[18],[20],[24],[32]$} \\
\hline Trustless and Transparent & To ensure the whole healthcare process is trustworthy and transparent. & {$[19],[20],[21],[22],[31]$} \\
\hline Complexity & To minimize the complexity for higher technology adoption. & {$[19],[20],[24],[33]$} \\
\hline
\end{tabular}

strongest obstacles in current healthcare management systems. One of the major reasons for this is the lack of universal standards for interoperability. Blockchain promises to empower EHR interoperability for the current healthcare systems [20], [24].

\section{B. Data Security:}

Another vital requirement while designing such blockchain healthcare systems is to secure the patients sensitive data. As there are multiple entities involved in the blockchain based healthcare mechanisms, the security should be at the prime focus. Blockchain is expected to ensure more security, privacy and trust as compared with traditional healthcare systems. One of the primary reason for more privacy and trust is because all the involved entities are aware of all the data transaction within the system [28], [29], [30].

\section{Data consistency/Integrity/Immutability:}

One of the challenges in current healthcare management systems is the fragmentation and inconsistency of the medical data. Data inconsistency may cause delay and higher costs to complete the overall healthcare process for any user. Therefore, a blockchain based healthcare system must ensure that the healthcare data is consistent and unfeasible to be altered by unauthorized entities [24], [29], [30].

\section{Cost/Resources Effectiveness:}

Current healthcare systems are consuming more resources in terms of cost, computations, time and physical resources. For example, in most of the transactions, there needs to be intermediates that may take more delay or resources to perform the specific tasks [20], [24]. One of the key requirements will be to reduce the transactions cost/delay that could be because of third-parties, multiple involved entities or for any other reasons.

\section{E. Trustless and Transparent:}

With the current healthcare system, pre-existing trust need to be developed among various stakeholders to ensure the safer data storing and sharing processes. As the data is stored and shared among various parties, maintaining complete trust and data transparency would be a big ask [21], [22],
[31]. A blockchain based healthcare system will eliminate the intermediates overhead, so it should built trustless and transparent healthcare systems.

\section{F. Complexity:}

Current multi-stakeholders based healthcare systems are relatively more complex in terms of storage, sharing and processing the medical data and other billing related information. Therefore, one of the requirements for a blockchainhealthcare system is to have less complex processes for the healthcare systems to avoid complexities and unnecessary delays at various phases [20].

\section{Potential Challenges}

Following, we identified some of the potential challenges for building blockchain based healthcare systems that need to be addressed properly before the actual deployment.

\section{A. Scalability Restrictions:}

One of the potential challenges in blockchain healthcare would be of the scalability. The trade-off between the available computing capabilities versus the amount of medical transactions could limit the scalability of such healthcare systems [32].

\section{B. High Development Cost:}

The blockchain based healthcare systems may take high development and operation costs. The government and healthcare sector still need to define the different kinds of development, operations and total deployment cost for all involved stakeholders. Thus, it is crucial to find the optimal ways to reduce the overall cost and resources for building such systems [20], [24].

\section{Standardization Challenges:}

For the successful deployment in healthcare applications, appropriate standards must be defined by standardization bodies $[9,19]$. For example, in the case of healthcare information stored on the blockchain, it should be made clear that what data, size and format can be sent to blockchain. Thus, it must be defined well what medical data is stored on or off the blockchain [20], [24], [32]. 


\section{Cultural Resistance:}

The current society is mostly habitual of such healthcare processes that are accessed either through paperwork based procedures or in some cases through online means such as EHR/EMR and other online health services [19, 24]. In the current time, the patients data is not so commonly shared with multiple parties. Therefore this cultural shift will be one of the major challenges as changing the behavior of the people towards data sharing in a distributed way will require some efforts [33].

\section{E. Regulatory Uncertainty:}

Regulatory bodies would face challenges in order to define the policies that will consider the collaboration of various stakeholders to draft a complete ecosystem that also takes into account the existing regulatory framework. The Health Insurance Portability and Accountability Act (HIPAA) [34] is also currently working on defining the standards to preserve the privacy of users medical records.

\section{F. Security and Privacy Concerns:}

Blockchain technology with healthcare data provides some security features [9, 14]. However, security and privacy are still the foremost concerns in blockchain based healthcare and require concrete security solutions. For example, an authorized entity should be able to access the data and data storage and sharing must be done in a secure way [28], [29], [30], [32].

\section{G. Unwillingness to Share:}

Some of the stakeholders providing healthcare services still hesitate to share the data with other parties. For example, hospitals and insurance payers do not want to share the data very easily to other entities [2], [3], [8]. One of the reasons for this can be that the hospital may want to keep the cost data to themselves and may set different costs for different patients. Hence, the trust must be developed between various entities so that each entity should agree to share the data for better healthcare systems.

\section{SMART CONTRACTS FOR BLOCKCHAin-AWARE HEALTHCARE SYSTEMS}

A Smart Contract is an integral part of the blockchain based applications. It is an agreement made among various involved parties in the defined system. A smart contract is a computer protocol that follows specific rules, codes and constraints agreed by all participants in the network. For example, a smart contract for banking transactions or financial purposes includes all the terms and conditions agreed by each stakeholder available in that process. The traditional contracts are considered to be lengthy and experienced as a resource consuming process that is made either in form of writing or any actions [15], [16]. In contrast, smart contracts are computer based digital programs which are self-executive when the required terms are fulfilled.

Healthcare systems enable a number of relevant parties to work collaboratively and efficiently for better and enhanced medical services. Therefore, defining appropriate rules in the smart contract for healthcare will be crucial and must include the consent of all the relevant parties. In the healthcare blockchain, the patient and other stakeholders in the network should set up their details and signed the agreement for accepting the terms in order to develop the requirements in the smart contract [35]. For example, which hospitals can store and share the patient data, which doctors can access and append the data and what kind of data is available for pharmacy and laboratory. Figure 2 highlights the use of smart contracts in blockchain based healthcare systems where multiple service providers are operating. Once the appointment between patient, healthcare providers and pharmacists specified in the contract takes place and the transaction containing information about the data arrives to the address of the smart contract, then the distributed virtual machine of the blockchain executes the programming code process. Some of the fundamental elements which smart contracts for the healthcare may contain is shown in figure 2, for example; Address specifies the address for the patient's data on the database and address itself can be stored in blockchain; Access - code is a number that denotes to whom the patient gives permission of accessing the information such as doctor or any third party like relatives and friends; state represents the variables or function in the system and code specifies the agreement in which stakeholders signed it and other function to be performed. Once they agreed the terms, then the transaction details will record in the system and also the other entities will receive the transaction information.

As compared with existing available contracts, a smart contract is faster as well as it also decreases the time for executing and deploying the patients data. Since it a decentralized system, no one can act as a patient or healthcare provider. This system is relatively more safe to protect any medical document. Hackers cannot be able to modify or edit the document without the patients permission. A Smart Contract can directly deal with the stakeholders, whose signature is in the legal agreement. A Smart contract is considered as more cost-efficient because it will use less resources and eliminate the additional costs. Smart contracts are more trustworthy by having the property of immutable which means no one can modify /edit or delete the patient data without the patient's permission and this can be automatically encrypt the protocol/rules. The Smart Contract simplifies the transaction happening in the Blockchain and makes it easier to perform [16], [35].

In addition to the useful advantages of building smart contracts, there are also needs to address some challenges for successful utilization of smart contracts in healthcare systems. For example, if the codes or rules are written wrong (having some error) then the process will make a mistake. Without programmable knowledge, its not easy to write or understand the algorithm and if the mistakes happen in the code, then it is very costly to fix a bug. There is also an issue with the legal framework in a smart contract as this is not validated by the government completely. A Smart Contract needs an invoking support to perform some tasks, meaning that it cannot perform automatically without any action or support [36]. 


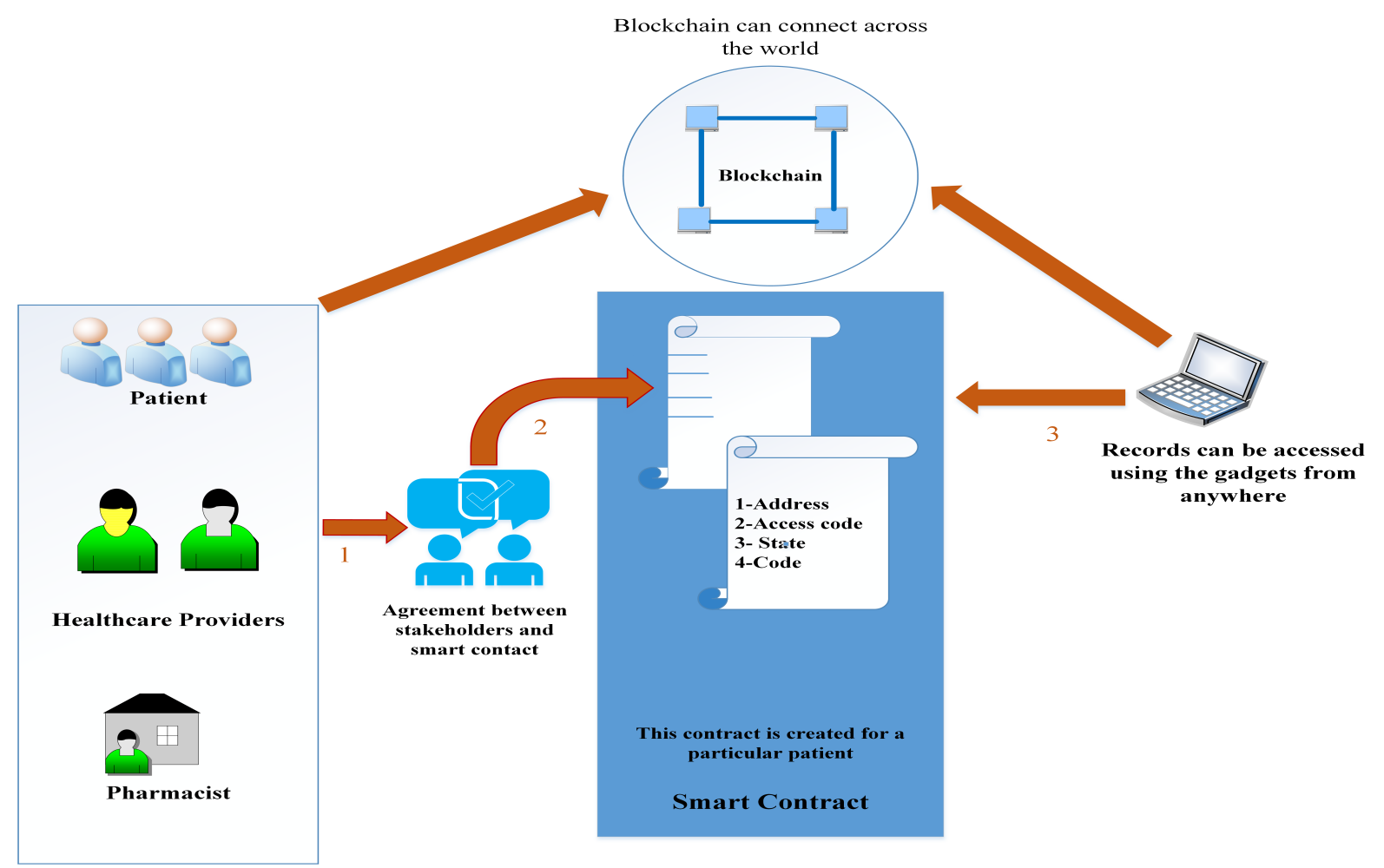

Fig. 2: Smart contract for healthcare scenario.

\section{Discussion And Future Scope}

The healthcare industry is facing digital revolution because of rapid developments in Information Communication Technology (ICT). The hype regarding the utilization of blockchain in the healthcare seems to be a reality in the coming future as researchers are exploring various aspects of blockchain healthcare systems. The top priority in healthcare landscape is to provide secure and safer means of accessing the patients's medical information throughout the whole process. Blockchain technology assumed to be among one of the suitable way of storing and sharing the medical information and by this only authorized entities are able to access the healthcare data. The potential of blockchain in healthcare is being realized by various involved stakeholders and its immense impact to improve the healthcare industry in terms of better medical services for the required patients and enhanced healthcare economy and revenues. One of the vital on-going obstacle in the current EHR systems is lack of nationwide healthcare interoperability and blockchain is the potential technology to handle this issue to some extent.

Apart from other questions, blockchain should address the the concerns regarding the storage of medical data. As blockchain itself is very restricted in terms of storing the actual healthcare data, so there is wider scope to propose concrete solutions for secure storage of the medical information. One solution for this is already available in the literature which offers to store the actual data in the server/database and the address of that data can be stored in the blockchain. The data retrieval or append and other operations can be done using that address which is stored in blockchain and linked with the database. In addition, concrete answers are also required for various security measures, for example what kind of security (symmetric/asymmetric) is more suitable for such healthcare applications and how things like key managements will be handled. Moreover, certain healthcare use cases require solutions that should be anonymous, transparent and repudiation.

The future of blockchain in the healthcare sector seems to be quite prominent and visionary. However, the practicality of the healthcare application using blockchain is mostly untested yet. But with the features that blockchain promises to add in the current healthcare systems would enable improved and better quality of healthcare services. Blockchain technology will empower the patients to take more control of their medical data and can handle their healthcare information in appropriate ways for managing overall health conditions. By this, an accurate, faster and improved data sharing healthcare mechanism could be drafted that can fulfill the needs of current healthcare requirements and also facilities the patients with the desired medical services.

\section{CONCLUSION}

With the recent advancements in internet and network technologies, there is a clear need of the enhancement in the quality of medical and healthcare services. There are numerous shortcomings in the current healthcare systems that seek solutions based on distributed and decentralized approaches. In this context, blockchain technology can play a leading role in providing the solutions that are decentralized and can ensure the security and integrity of the medical information. Therefore, the main focus of this work is to provide an 
overview of blockchain technology in the healthcare sector. This article identifies key application areas in the healthcare domain where blockchain technology can be a useful addition. Moreover, various requirements and challenges for blockchain based healthcare systems are presented in this work. Finally the concept of smart contracts for blockchain based healthcare systems is highlighted.

\section{ACKNOWLEDGMENT}

This work was supported by TEKES Finland project Towards Digital Paradise and by Academy of Finland under projects Industrial Edge and 6Genesis Flagship (grant 318927) projects. The authors would also like to acknowledge the contribution of the COST Action CA15127 (RECODIS) and CA16226 (SHELD-ON).

\section{REFERENCES}

[1] T. Aste, P. Tasca, and T. D. Matteo, Blockchain technologies: The foreseeable impact on society and industry, Computer, vol. 50, no. 9, pp. 1828, 2017.

[2] R. Beck, Beyond bitcoin: The rise of blockchain world, Computer, vol. 51, no. 2, pp. 5458, February 2018.

[3] M. Mettler, "Blockchain technology in healthcare: The revolution starts here," 2016 IEEE 18th International Conference on e-Health Networking, Applications and Services (Healthcom), Munich, 2016, pp. 1-3.

[4] S. B. Baker, W. Xiang, and I. Atkinson, Internet of things for smart healthcare: Technologies, challenges, and opportunities, IEEE Access, vol. 5, pp. 2652126 544, 2017.

[5] I. Ahmad, T. Kumar, M. Liyanage, J. Okwuibe, M. Ylianttila and A. Gurtov, "Overview of 5G Security Challenges and Solutions," in IEEE Communications Standards Magazine, vol. 2, no. 1, pp. 36-43, MARCH 2018.

[6] T. Kumar, M. Liyanage, I. Ahmad, et al. User privacy, identity and trust in 5G. In: A Comprehensive Guide to 5G Security. Hoboken: Wiley, 2018.

[7] K.Peterson, Kevin, et al. "A blockchain-based approach to health information exchange networks." In Proc. NIST Workshop Blockchain Healthcare, vol. 1, pp. 1-10. 2016.

[8] C. Esposito, A. De Santis, G. Tortora, H. Chang and K. K. R. Choo, "Blockchain: A Panacea for Healthcare Cloud-Based Data Security and Privacy?," in IEEE Cloud Computing, vol. 5, no. 1, pp. 31-37, Jan./Feb. 2018.

[9] Azaria A, Ekblaw A, Vieira T, Lippman A. MedRec: using blockchain for medical data access and permission management. International Conference on Open and Big Data (OBD). Vienna, Austria: IEEE; 2016:2530.

[10] T. Kumar, A. Braeken, M. Liyanage and M. Ylianttila, "Identity privacy preserving biometric based authentication scheme for Naked healthcare environment," 2017 IEEE International Conference on Communications (ICC), Paris, 2017, pp. 1-7.

[11] T. Kumar, M. Liyanage, A. Braeken, I. Ahmad and M. Ylianttila, "From gadget to gadget-free hyperconnected world: Conceptual analysis of user privacy challenges," 2017 European Conference on Networks and Communications (EuCNC), Oulu, 2017, pp. 1-6.

[12] Peng Zhang, Michael A. Walker, Jules White, Douglas C. Schmidt, Metrics for Assessing Blockchain-based Healthcare Decentralized Apps, 2017 IEEE 19th International Conference on e-Health Networking, Applications and Services (Healthcom), 2017.

[13] Yue, X., Wang, H., Jin, D., Li, M.,Jiang, W. (2016). Healthcare data gateways: found healthcare intelligence on Blockchain with novel privacy risk control. Journal of Medical Systems, 40(218).

[14] Yli-Huumo, J., Ko, D., Choi, S., Park, S., Smolander, K. (2016). Where is current research on blockchain technology?a systematic review. PloS one, 11(10)

[15] Massimo Bartoletti and Livio Pompianu,"An empirical analysis of smart contracts: platforms,applications, and design patterns",Universita degli studi di Cagliari, 2017.

[16] C. Wright and A. Serguieva, "Sustainable blockchain-enabled services: Smart contracts," 2017 IEEE International Conference on Big Data (Big Data), Boston, MA, 2017, pp. 4255-4264.
[17] X. Liang, J. Zhao, S. Shetty, J. Liu and D. Li, "Integrating blockchain for data sharing and collaboration in mobile healthcare applications," 2017 IEEE 28th Annual International Symposium on Personal, Indoor, and Mobile Radio Communications (PIMRC), Montreal, QC, 2017, pp. $1-5$.

[18] Kuo, Tsung-Ting, Hyeon-Eui Kim, and Lucila Ohno-Machado. "Blockchain distributed ledger technologies for biomedical and health care applications." Journal of the American Medical Informatics Association 24.6 (2017): 1211-1220.

[19] Huihui Yang,Bian yang,"A Blockchain-based Approach to the Secure Sharing of Healthcare Data."Journal of Norwegian University of science and technology, 2018.

[20] RJ Krawiec, Dan Housman, Mark White, Mariya Filipova, Florian Quarre, Dan Barr, Allen Nesbitt, Kate Fedosova, Jason Killmeyer, Adam Israel, Lindsay Tsai , Blockchain : Opportunities for health care Deloitte, 2016.

[21] Nugent, Timothy and Upton, David and Cimpoesu, Mihai "Improving data transparency in clinical trials using blockchain smart contracts",F1000Research, vol.5,2016.

[22] Benchoufi, Mehdi, Raphael Porcher, and Philippe Ravaud. Blockchain Protocols in Clinical Trials: Transparency and Traceability of Consent. F1000Research 6 (2017): 66. PMC. Web. 2 May 2018.

[23] enchoufi, Mehdi, and Philippe Ravaud. Blockchain Technology for Improving Clinical Research Quality. Trials 18 (2017): 335. PMC. Web. 2 May 2018.

[24] Chet Stgnaro,"White Paper: Innovative Blockchain Uses in Healthcare",Freed Associates, 2017.

[25] Clauson, Kevin A., et al. "Leveraging Blockchain Technology to Enhance Supply Chain Management in Healthcare." Blockchain in Healthcare Today (2018).

[26] Clauson, Engelhardt, Mark A. "Hitching healthcare to the chain: An introduction to blockchain technology in the healthcare sector." Technology Innovation Management Review 7, no. 10 (2017).

[27] Boulos, M. N. K., Wilson, J. T., Clauson, K. A. (2018). Geospatial blockchain: promises, challenges, and scenarios in health and healthcare, International Journal of Health Geographics, 17:25, 2018.

[28] M. Puppala, T. He, X. Yu, S. Chen, R. Ogunti, and S. T. C. Wong, Data security and privacy management in healthcare applications and clinical data warehouse environment, in 2016 IEEE-EMBS International Conference on Biomedical and Health Informatics (BHI), Feb 2016 , pp. 58

[29] Al Omar, A., Rahman, M. S., Basu, A., Kiyomoto, S. (2017). Medibchain: A blockchain based privacy preserving platform for healthcare data. In International conference on security, privacy and anonymity in computation, communication and storage (pp. 534543).

[30] G. Zyskind, O. Nathan and A. '. Pentland, 'Decentralizing Privacy: Using Blockchain to Protect Personal Data," 2015 IEEE Security and Privacy Workshops, San Jose, CA, 2015, pp. 180-184.

[31] Q. Xia, E. B. Sifah, K. O. Asamoah, J. Gao, X. Du and M. Guizani, "MeDShare: Trust-Less Medical Data Sharing Among Cloud Service Providers via Blockchain,” in IEEE Access, vol. 5, pp. 14757-14767, 2017.

[32] Laure A.Linn, Martha B.Koo, Blockchain for health data and its potential use in health IT and health care related research,2016.

[33] Begley, Robert. "Information Records Management and Blockchain Technology: Understanding its Potential.” PhD diss., Northumbria University, 2017.

[34] P. F. Edemekong, M. J. Haydel, (2018). Health Insurance Portability and Accountability Act (HIPAA). In StatPearls [Internet]. StatPearls Publishing.

[35] Simon Janin, Smart contract in healthcare Health Management.org (Vol. 18, issue 1), 2018.

[36] Dr. Maciej Hulicki, The legal framework and challenges of smart contracts applications, Cardinal Wyszynski University of Warsaw, 2017. 